\title{
Perfil da mortalidade de centenários em Curitiba - Paraná, 2010-2019
}

Mortality profile of centennials in Curitiba - Paraná, 2010-2019

\author{
Perfil de mortalidad de los centenarios en Curitiba - Paraná, 2010-2019
}

Gustavo Fernandes de Souza1*, João Guilherme Bernart Sernajotto ${ }^{1}$, Gabriela Baby Litvinski ${ }^{1}$, Matheus Henrique Gonzatti ${ }^{1}$, Nilza Teresinha Faoro², Sandra Lúcia Vieira Ulinski ${ }^{3}$, Solena Ziemer Kusma Fidalski ${ }^{1}$.

\section{RESUMO}

Objetivo: Estabelecer o perfil de mortalidade de centenários no município de Curitiba-PR. Métodos: Tratase de um estudo epidemiológico descritivo, retrospectivo, a partir dos óbitos de idosos na faixa etária de 100 anos ou mais, residentes no município de Curitiba. Os dados secundários foram coletados no Sistema de Informação de Mortalidade (SIM). As variáveis consideradas foram idade, sexo, raça/cor, escolaridade, ocupação habitual, local de ocorrência do óbito, causas básicas do óbito (CID-10), de 2010 a 2019. A análise dos dados foi descritiva por meio de distribuição de frequências. Resultados: No banco de dados, constavam 498 óbitos de centenários e a idade variou de 100 a 115 anos. As principais causas de óbito foram doenças do aparelho circulatório $(32,7 \%)$, do aparelho respiratório $(21,3 \%)$, do sistema nervoso (12\%), neoplasias $(7,2 \%)$ e causas externas $(6,8 \%)$. Para as mulheres, as principais causas foram doenças do aparelho circulatório $(37 \%)$ e, para os homens, destacaram-se as doenças do aparelho respiratório (29,8\%). Conclusão: $O$ perfil de mortalidade entre os centenários de Curitiba identificou que predominaram as mulheres, donas de casa, de cor branca, com escolaridade entre 4 e 11 anos e com uma média de 101 anos, sendo doenças cardiovasculares a principal causa de morte.

Palavras-chave: Epidemiologia, Idoso, Mortalidade.

\begin{abstract}
Objective: Establish the mortality profile of centenarians in the city of Curitiba-PR. Methods: This is a descriptive, retrospective epidemiological study, based on the deaths of elderly people aged 100 years and over, residing in the city of Curitiba. Secondary data were collected in the Mortality Information System (SIM). The variables considered were age, sex, race/color, education, usual occupation, place of death, primary causes of death (ICD-10), from 2010 to 2019. Data analysis was descriptive, through frequency distribution. Results: The database contained 498 deaths of centenarians and ages ranged from 100 to 115 years. The main causes of death were diseases of the circulatory system $(32.7 \%)$, the respiratory system $(21.3 \%)$, the nervous system (12\%), neoplasms (7.2\%) and external causes (6.8\%). Among women, the main causes were diseases of the circulatory system (37\%) and, for men, respiratory diseases stood out $(29.8 \%)$. Conclusion: The mortality profile among the centenarians of Curitiba identified women, housewives, white, with between 4 and 11 years of schooling, with an average of 101 years, predominated, and cardiovascular diseases are the main cause of death.
\end{abstract}

Keywords: Epidemiology, Elderly, Mortality.

${ }^{1}$ Universidade Positivo (UP), Curitiba - PR. *E-mail: gus.fernandes@hotmail.com

2 Pontifícia Universidade Católica do Paraná (PUC-PR), Curitiba - PR.

${ }^{3}$ Faculdades Integradas Norte do Panará (UNOPAR), Londrina - PR.

SUBMETIDO EM: 10/2021

ACEITO EM: 10/2021

PUBLICADO EM: 11/2021 


\section{RESUMEN}

Objetivo: Establecer el perfil de mortalidad de los centenarios en la ciudad de Curitiba-PR. Métodos: Se trata de un estudio epidemiológico descriptivo, retrospectivo, basado en la muerte de personas de 100 años o más, residentes en la ciudad de Curitiba. Los datos secundarios se obtienen del Sistema de Información de Mortalidad (SIM). Las variables consideradas fueron edad, sexo, raza / color, educación, ocupación, lugar de muerte, causas subyacentes de muerte (CIE-10), de 2010 a 2019. El análisis de los datos fue descriptivo a través de la distribución de frecuencias. Resultados: La base de datos contiene 498 muertes de centenarios y las edades oscilaron entre 100 y 115 años. Las principales causas de muerte fueron enfermedades del sistema circulatorio $(32,7 \%)$, respiratorio $(21,3 \%)$, sistema nervioso $(12 \%)$, neoplasias $(7,2 \%)$ y causas externas $(6,8 \%)$. Para las mujeres, las principales causas fueron las enfermedades del sistema circulatorio (37\%) y, para los hombres, se destacaron las enfermedades respiratorias $(29,8 \%)$. Conclusión: El perfil de mortalidad entre centenarios en Curitiba identificó que predominaban mujeres, amas de casa, blancas, con escolaridad entre 4 y 11 años y con un promedio de 101 años, y las enfermedades cardiovasculares son la principal causa de muerte.

Palabras clave: Epidemiología, Anciano, Mortalidad.

\section{INTRODUÇÃO}

De acordo com o Estatuto do Idoso, no Brasil, define-se como idoso qualquer pessoa com idade igual ou superior a 60 anos (BRASIL, 2009). Essa população apresenta um crescimento significativo, impactando 0 modo de viver e a forma como a sociedade se organiza (MARTINS SC, et al., 2018).

O perfil demográfico da população vem se modificando expressivamente devido ao aumento da esperança média de vida e da redução da taxa de natalidade, resultando no envelhecimento global da população (MARTINS SC, et al., 2018).

Uma vida mais longa é uma realidade em praticamente todas as partes do mundo. A velocidade de envelhecimento da população é muito mais rápida atualmente. Uma criança que nasceu no Brasil em 2015, por exemplo, poderá viver 20 anos mais do que uma nascida há 50 anos (ORGANIZAÇÃO MUNDIAL DA SAÚDE, 2015).

De acordo com projeções do Instituto Paranaense de Desenvolvimento Econômico e Social (IPARDES), a partir do final dos anos 2030, o número de idosos irá superar o número de crianças, no Paraná. Os valores passarão de 32 idosos para cada grupo de 100 crianças, no ano de 2010, para 118 idosos para cada 100 crianças, em 2040 (INSTITUTO NACIONAL DO CÂNCER, 2019).

Em várias regiões do mundo, tem-se observado um aumento expressivo do número de idosos e em especial da população de centenários, pessoas com 100 anos ou mais. Esse aumento tem chamado a atenção de pesquisadores (REIS CS e TURRA CM, 2016). Segundo relatório da Organização das Nações Unidas (ONU) do ano de 2019, citado em Nepomuceno MR e Turra CM (2020), evidenciou-se que, nos últimos 25 anos, o número de centenários que vivem no mundo aumentou cinco vezes, atingindo mais de 410.000 pessoas, em 2015. Neste sentido, observa-se maior concentração de populações idosas em regiões de baixa mortalidade.

Em 2015, o Revision of the World Population Prospects publicou uma estimativa de que, em 1990, havia 96.000 centenários no mundo. Tal número alcançou 125.000 indivíduos, em 1995, e 160.000, em 2000. Em 2015, atingiu 451.000 centenários. Isso demonstra que o número global de centenários tem aumentado muito mais rápido ao longo do tempo. Este relatório também evidenciou que o número de centenários é muito pequeno em países de baixa renda, representando menos de $1 \%$ do total mundial (ROBINE JM e CUBAYENES S, 2017).

No Brasil, a população centenária cresceu duas vezes e meia entre 1960 e 2000 e, atualmente, compreende mais de 24.000 indivíduos, dos quais $70 \%$ são mulheres, comprovando a tendência mundial da 
feminização dos idosos centenários. Observa-se também que a população brasileira está envelhecendo mais rápido em regiões mais ricas (NEPOMUCENO MR e TURRA CM, 2020).

Nas últimas décadas, o que se tem observado, no Brasil, é uma diminuição da mortalidade por doenças infectocontagiosas e aumento expressivo de óbitos decorrentes de doenças crônicas não transmissíveis, sendo o número de óbitos destas últimas, maior que os de doenças infectocontagiosas. Essa mudança, apesar de evidenciar o desenvolvimento do país, traz consigo dificuldades em especial para os sistemas de Saúde e da Previdência Social, em função de uma carga elevada de custos financeiros devido especialmente a mortalidade e invalidez (OLIVEIRA TC, et al., 2015).

No grupo de centenários, o perfil de mortalidade varia quando comparado aos não idoso ou idosos com menos de 80 anos. Grande parte dessa diferença decorre das comorbidades mais prevalentes que os centenários possuem, além da forma como a morte é enfrentada nessa faixa etária (CARVALHO MHR, et al., 2014).

Os centenários são um grupo populacional em crescimento, no entanto, poucos estudos exploram características ou informações sobre essa população. Devido as suas diferenças quando comparados a outros grupos, não é correto extrapolar o resultado de estudos realizados com pessoas mais jovens (MARTINS SC, et al., 2018). A maioria dos estudos não considera os indivíduos com 100 anos ou mais como um grupo separado, muitas vezes sendo agrupado no grupo dos idosos com 80 anos ou mais (EVANS CJ, et al., 2014).

Sendo assim, a caracterização, bem como a interpretação do perfil da mortalidade na população centenária brasileira, assim como do município de Curitiba, pode contribuir para o planejamento de ações públicas. Nesse sentido, o objetivo deste estudo foi estabelecer o perfil de mortalidade de centenários no município de Curitiba-PR, no período de 2010 a 2019, determinando o perfil da mortalidade de acordo com o sexo, raça, escolaridade, local de ocorrência e causa básica de óbito, a fim de melhor entender o panorama de mortalidade desta faixa etária e de subsidiar programas e ações em saúde direcionadas a essa população.

\section{MÉTODOS}

Estudo epidemiológico descritivo, retrospectivo, a partir dos óbitos de idosos na faixa etária com 100 anos ou mais, residentes no município de Curitiba, capital do estado do Paraná, localizada na região sul do Brasil.

Os dados secundários foram coletados no Sistema de Informação de Mortalidade (SIM) da Secretaria Municipal da Saúde de Curitiba. As variáveis consideradas foram idade, sexo, raça/cor, escolaridade, ocupação habitual, local de ocorrência do óbito, causas básicas do óbito de acordo com os capítulos da Classificação Internacional de Doenças (CID-10), com recorte temporal de primeiro de janeiro de 2010 a 31 de dezembro de 2019.

Foi realizada análise descritiva por meio de distribuição de frequências através do programa Epi Info versão 7.2. O teste do qui-quadrado foi utilizado para verificar diferenças de proporções entre as variáveis categóricas. Foi considerado como significativo o valor de $p$ menor que 0,05.

Quanto às considerações éticas, o projeto obteve sua aprovação junto ao Comitê de ética e pesquisa (CEP) da Universidade Positivo sob Certificado de Apresentação de Apreciação Ética (CAAE) de n. 31260620.3.0000.0093 em maio de 2020; e junto ao CEP da Secretaria Municipal da Saúde de Curitiba sob CAAE de n. 31260620.3.3001.0101 em junho de 2020.

\section{RESULTADOS}

O banco de dados é composto por um $\mathrm{N}$ de 498 idosos que foram a óbito com idade igual e/ou acima de 100 anos, no período de 2010 a 2019 em Curitiba, perfazendo uma média de 49 óbitos ao ano. Neste perfil, predominaram as mulheres $(77,1 \%)$, de cor branca $(92,2 \%)$, com escolaridade entre 4 e 11 anos $(47 \%)$. Em relação à idade, a média foi de 101 anos, variando de 100 e 115 anos. Para a ocupação, predominaram as donas de casa com $50,1 \%$, seguidas pelos aposentados (15,7\%) e pelos trabalhadores rurais $(5,8 \%)$. 
Tabela 1 - Características dos indivíduos com mais de 100 anos que foram a óbito de 2010 a 2019.

\begin{tabular}{|c|c|c|}
\hline Variável & $\mathbf{N}$ & $\%$ \\
\hline \multicolumn{3}{|l|}{ Sexo } \\
\hline Feminino & 384 & $77,1 \%$ \\
\hline Masculino & 114 & $22,9 \%$ \\
\hline \multicolumn{3}{|l|}{ Raça } \\
\hline Amarela & 5 & $1,1 \%$ \\
\hline Branca & 427 & $92,2 \%$ \\
\hline Parda & 17 & $3,67 \%$ \\
\hline Preta & 14 & $3,02 \%$ \\
\hline \multicolumn{3}{|l|}{ Escolaridade } \\
\hline Nenhuma & 99 & $19,9 \%$ \\
\hline 1 a 3 anos & 85 & $17,1 \%$ \\
\hline 4 a 7 anos & 131 & $26,3 \%$ \\
\hline 8 a 11 anos & 103 & $20,7 \%$ \\
\hline $12 \mathrm{e}$ mais & 50 & $10,0 \%$ \\
\hline Ignorado & 30 & $6,0 \%$ \\
\hline \multicolumn{3}{|l|}{ Local de ocorrência } \\
\hline Domicilio & 187 & $37,6 \%$ \\
\hline Hospital & 249 & $50,0 \%$ \\
\hline Outros & 26 & $5,2 \%$ \\
\hline Outros estabelecimentos de saúde & 36 & $7,2 \%$ \\
\hline \multicolumn{3}{|l|}{ Causa básica do óbito } \\
\hline Doenças infecciosas e parasitárias & 9 & $1,8 \%$ \\
\hline Neoplasias & 36 & $7,2 \%$ \\
\hline Doenças do sangue e órgãos hematopoiéticos & 2 & $0,4 \%$ \\
\hline Doenças endócrinas, nutricionais e metabólicas & 22 & $4,4 \%$ \\
\hline Transtornos mentais e comportamentais & 7 & $1,4 \%$ \\
\hline Doenças do sistema nervoso & 60 & $12,0 \%$ \\
\hline Doenças do aparelho circulatório & 163 & $32,7 \%$ \\
\hline Doenças do aparelho respiratório & 106 & $21,3 \%$ \\
\hline Doenças do aparelho digestivo & 11 & $2,2 \%$ \\
\hline Doenças da pele e do tecido subcutâneo & 3 & $0,6 \%$ \\
\hline Doenças do sistema osteomuscular & 2 & $0,4 \%$ \\
\hline Doenças do aparelho geniturinário & 33 & $6,6 \%$ \\
\hline Sintomas, sinais e achados anormais no exame clínico & 10 & $2,0 \%$ \\
\hline Causas externas & 34 & $6,8 \%$ \\
\hline Total & 498 & 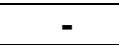 \\
\hline
\end{tabular}

Fonte: Souza GF, et al., 2021.

As principais causas de óbito entre os idosos com mais de 100 anos foram doenças do aparelho circulatório $(32,7 \%)$, do aparelho respiratório $(21,3 \%)$, do sistema nervoso (12\%), neoplasias $(7,2 \%)$ e causas externas $(6,8 \%)$. Entre as mulheres, as principais causas foram doenças do aparelho circulatório (37\%) seguidas pelas doenças do aparelho respiratório (18,8\%). Para os homens, essas causas se invertem nas quais $29,8 \%$ faleceram por doenças do aparelho respiratório e 18,4\% por doenças aparelho circulatório.

As doenças do aparelho circulatório foram a principal causa de óbito em todos os anos no período de 2010 a 2019 ( $p=0,04)$. Identificou-se que 61,3\% dos óbitos por doenças respiratórias ocorreram em hospital, enquanto $47,9 \%$ dos óbitos por doenças circulatórias ocorreram no domicílio $(p<0,05)$. Entre os óbitos por neoplasias, $52,8 \%$ foram a óbito no hospital $(p<0,05)$

Foi possível observar iniquidades entre os indivíduos com relação à escolaridade. A maior parcela de indivíduos com 12 anos ou mais de estudo foi de 95,7\% para a raça branca e 4,3\% para a raça preta.

Quanto ao local de ocorrência, $50 \%$ foram a óbito em ambiente hospitalar, seguido do domicílio (37,6\%). No entanto, comparando-se o percentual de óbitos em domicílios entre mulheres e homens, o percentual das mulheres excede em relação aos homens $(p<0,05)$ (Tabela 2). 
Tabela 2 - Percentual de óbitos dos indivíduos com mais de 100 anos em Curitiba, de 2010 a 2019.

\begin{tabular}{ccccc}
\hline \multirow{2}{*}{ Sexo } & Domicílio & Hospital & Outros & $\begin{array}{c}\text { Outros estabelecimentos de } \\
\text { saúde }\end{array}$ \\
\cline { 2 - 5 } & $152(39,6 \%)$ & $179(46,6 \%)$ & $25(6,5 \%)$ & $28(7,3 \%)$ \\
\hline Feminino & $35(30,7 \%)$ & $70(61,4 \%)$ & $1(0,9 \%)$ & $8(7,0 \%)$ \\
\hline Masculino & $187(37,6 \%)$ & $249(50 \%)$ & $26(5,2 \%)$ & $36(7,2 \%)$ \\
\hline Total & & & &
\end{tabular}

Fonte: Souza GF, et al., 2021.

\section{DISCUSSÃO}

O presente estudo evidenciou que, de acordo com o SIM de Curitiba, no período de primeiro de Janeiro de 2019 a 31 de Dezembro de 2019, ocorreram 498 óbitos de pessoas com 100 anos ou mais. Desse total, 114 eram do sexo masculino e 384 do sexo feminino. O perfil de mortalidade entre os centenários de Curitiba identificou que predominaram as mulheres, donas de casa, de cor branca, com escolaridade entre 4 e 11 anos com uma média de 101 anos. As principais causas de morte foram as doenças cardiovasculares seguidas pelas respiratórias.

A feminização do envelhecimento em Curitiba, identificada nesse estudo, acompanha uma tendência já evidenciada no Brasil e no mundo. Estudos demonstram que as mulheres contemplam a maior parcela da população idosa em todas as regiões do mundo, com estimativas de que as mulheres vivam, aproximadamente, de cinco a sete anos a mais que os homens (EVANS CJ, et al., 2014; YU R, et al., 2017; MAXIMIANO-BARRETO MA, et al., 2019; CAMPOS VS, 2020).

As características das populações centenárias variam em diferentes países, mas estudos como o de Yu R, et al. (2017), mostra que mais de $85 \%$ dos idosos, em Hong Kong, eram do sexo feminino, e também em Evans CJ, et al. (2014), em que mais de $86 \%$ dos idosos do Reino Unido eram do sexo feminino, o que demonstra uma predominância do gênero feminino e que as mulheres são mais longevas que os homens (EVANS CJ, et al., 2014; YU R, et al., 2017).

O predomínio das mulheres na terceira idade está associado a aspectos biológicos e a não exposição a fatores de risco de mortalidade. Vários fatores, de forma isolada ou concomitante, determinam que as mulheres possuam uma expectativa de vida maior que os homens, como por exemplo: inclusão diferenciada no mercado de trabalho; exposição ao tabagismo e ao uso de álcool; a diferença de atitude em relação à saúde/doença, pois, contrariamente aos homens, a mulher normalmente demonstra preocupação com prevenção, informação e cuidados de saúde. Portanto, é possível dizer que o maior cuidado feminino com a saúde repercute de maneira positiva em atingir faixa etária mais elevada. A falta desses cuidados, associada à maior exposição a riscos, por outro lado, repercute negativamente na longevidade do sexo masculino (MAXIMIANO-BARRETO MA, et al., 2019; CAMPOS VS, 2020; MOURA ECD, et al., 2016).

Em 1998, uma mulher, ao completar 60 anos, poderia esperar viver 2,8 anos a mais em relação aos homens. Em 2008, esse número saltou para 3,2 anos. Esses dados trazem consigo uma reflexão sobre a qualidade dessa diferença de expectativa média de vida, uma vez que com o aumento da sobrevida saudável e ativa feminina, há também aumento da longevidade com qualidade de vida ruim e incapacidade (CAMARGOS MCS e GONZAGA MR, 2015).

Metade dos óbitos registrados dos centenários, em Curitiba, ocorreram em hospitais e não em âmbito domiciliar. Isto se deve provavelmente à cultura da institucionalização do processo de óbito, decorrente de um incremento técnico da área da saúde, disponibilização de leitos e hospital exclusivo para internação de idosos, reflexo do desenvolvimento social e econômico do município de Curitiba. Isto melhorou o acesso à atenção especializada, possibilitando que mais pessoas recebessem os cuidados ao final da vida em ambiente hospitalar (MARCUCCI FCI e CABRERA MAS, 2015).

Por outro lado, a disponibilidade de suporte de Cuidados Paliativos tem sido vista fortemente associada com a possibilidade de ocorrência dos óbitos em domicílio, permitindo com que ocorra uma maior autonomia de escolha para os pacientes e também para seus cuidadores, estabelecendo-se como foco a qualidade de 
vida durante os períodos prévios ao falecimento. Apesar disso, fatores como a dificuldade do suporte em saúde domiciliar, a menor aceitação da família e o fato de ser mais difícil o manejo da dor e controle dos sintomas também limitam o óbito em domicílio (MARCUCCI FCI e CABRERA MAS, 2015).

Essa não é a realidade de muitos locais no mundo, como em Taiwan, onde $57,7 \%$ dos óbitos de centenários foram domiciliares, e no Reino Unido onde $45,1 \%$ dos óbitos dos centenários ocorreram em suas casas. Apesar do envelhecimento ser um fenômeno global, há diferenças regionais, de acordo com a cultura, etnia, educação e outros e tais variáveis influenciam no local de ocorrência do óbito. Em alguns países, é culturalmente aceito que a morte ocorra em suas residências, quando possível. Em outras ocasiões, essa opção se dá por políticas locais de transferência ou então pela possibilidade financeira de países darem uma assistência adequada em domicílio (EVANS CJ, et al., 2014; CHEN Y-C, et al., 2019).

A principal causa de morte dos centenários, em Curitiba, foram as doenças circulatórias, as quais também correspondem a principal causa de óbito em idosos na faixa etária a partir dos 60 anos, de acordo com estatísticas nacionais. Além disso, desde a década de 60, as doenças do aparelho circulatório representam a principal causa de mortalidade do brasileiro, trazendo consigo grandes impactos para o sistema de saúde (OLIVEIRA GMM, et al., 2020).

Em Curitiba, as doenças do aparelho circulatório representaram um terço dos óbitos totais, porém com uma diferença entre os sexos: enquanto as mulheres faleceram mais por doenças cardiovasculares, para os homens predominaram as doenças respiratórias, seguido pelas causas circulatórias. Este cenário é diferente quando comparado com os Estados Unidos, por exemplo, em que, entre 2000 e 2014, a principal causa de morte foram as doenças cardiovasculares de forma equivalente em ambos os sexos ( $X U$ J, 2016).

A situação apresentada, em nosso estudo, abre a possibilidade a duas inferências: primeiro, pelo número total de mulheres centenárias, no estudo, ser superior ao de homens, a média entre os sexos tende a ser mais condizente com as causas femininas, apresentando que a principal causa geral de morte entre os centenários são causas circulatórias. Segundo, é que a mortalidade por doenças respiratórias em homens pode ser reflexo de um histórico de tabagismo que, nas décadas de 1950/1960, era majoritariamente masculino, sendo que mulheres não tinham esse hábito socialmente aceito, o que resulta no quadro atual de mortalidade entre os centenários homens (INSTITUTO PARANAENSE DE DESENVOLVIMENTO ECONÔMICO E SOCIAL, 2020).

A segunda causa de óbito geral entre os centenários de Curitiba foi a respiratória $(21,3 \%)$. As causas de óbitos por doenças respiratórias na população de centenários podem estar associadas a maior quantidade de indivíduos acamados nessa faixa etária. Pessoas nessas condições apresentam maior risco de desenvolverem complicações decorrentes da fraqueza muscular diafragmática e intercostal, o que se associa com maior dificuldade para eliminar secreções ao não produzirem tosse efetiva e pela diminuição da função ciliar, o que acaba resultando no acúmulo de secreção nos pulmões e predispõe a quadros de infecções respiratórias e atelectasias (OLIVEIRA TC, et al., 2015).

Apesar disso, diferentemente de Curitiba em que as doenças respiratórias foram a segunda causa de morte, no estudo realizado em Hong Kong as causas respiratórias foram a principal causa de mortalidade $(39,8 \%)$ (YU R, et al., 2017). Uma explicação para essa diferença pode ser o Programa Nacional de Imunização Brasileiro. Para a prevenção dos óbitos por causas respiratórias, uma das principais medidas é o controle das infecções respiratórias através da imunização com a vacina contra o vírus Influenza, que contribuiu de forma significativa para a diminuição da mortalidade por causas respiratórias e dos gastos com internamentos. No Brasil, a vacinação contra a gripe é focada aos idosos e pessoas com comorbidades, sendo esses os grupos que apresentam maior risco de complicações da gripe. A vacinação atinge $70 \%$ de cobertura em todo o território nacional e demonstra forte evidência da sua efetividade (OLIVEIRA TC, et al., 2015).

Em Curitiba, $12 \%$ dos óbitos ocorreram por doenças do sistema nervoso nos centenários, ocupando o terceiro lugar na causa de morte nessa faixa etária. Diferentemente do que ocorreu nos Estados Unidos, em que as doenças relacionadas ao Alzheimer, entre 2000 e 2014, representaram 8,5\% dos óbitos entre os centenários (XU J, 2016). 
Ainda entre as causas de óbitos, as neoplasias ocuparam o quarto lugar, com $7,2 \%$ das mortes. Ao analisar o perfil de mortalidade, as neoplasias se apresentam como a segunda causa de óbito na maioria dos municípios brasileiros, havendo localidades em que é a causa número um. Quando se fala no perfil de saúde pública do Brasil, as neoplasias ocupam papel de destaque e vários fatores têm contribuído para um aumento da mortalidade por esta causa como, por exemplo, o aumento da expectativa de vida, modificações do estilo de vida, além de questões como o avanço no diagnóstico de câncer o a melhora da qualidade do registro de óbitos (RIBEIRO TS, et al., 2020).

Por outro lado, isto não ocorreu entre os centenários em outros países quando comparado a outros estudos em que o óbito por neoplasia foi de apenas aproximadamente 4\% (EVANS CJ, et al., 2014; IPARDES, 2020; $\mathrm{XU} \mathrm{J}, 2016$ ). Isto se deve muito provavelmente pelo menor número de exames de rastreamento realizados na população centenária em relação às demais faixas etárias. Além disso, a evolução de técnicas e novas políticas de cuidados paliativos que prolongam a vida possibilitam que os centenários vão a óbito por outras comorbidades e não pela neoplasia (CAPRIO AJ, 2016).

Quanto aos fatores de risco, ressalta-se que os riscos ocupacionais podem ser considerados fatores de risco positivos para mortalidade precoce, uma vez que interferem significativamente na saúde de muitos profissionais, podendo provocar moléstias, muitas vezes fatais ou incapacitantes, como o câncer de pele em trabalhadores rurais ou doenças pulmonares em profissionais que manuseiam produtos tóxicos (CEZAR-VAZ MR, et al., 2015; GAUDIANO J, et al., 2020). Assim, é possível inferir que a falta de riscos ocupacionais poderia ser um fator protetor para mortalidade precoce. Considerando os óbitos analisados, metade deles foram de idosos cuja ocupação era "dono de casa", o que permite dizer que a ocupação pode ter sido tal fator positivo para que eles chegassem à centenaridade.

Com relação às limitações do estudo, os resultados encontrados revelam um panorama da cidade de Curitiba, não necessariamente podendo ser estendido para outros cenários populacionais. Além disso, a literatura carece de estudos direcionados ao perfil de mortalidade dos centenários, afetando análises comparativas mais aprofundadas.

\section{CONCLUSÃO}

O País passa por um processo de envelhecimento da população. O número de centenários vem aumentando no Brasil e no mundo, tornando-se imperativo o estudo do perfil dessa população a fim de proporcionar serviços de saúde bem-preparados para o seu cuidado e também melhorar a qualidade de vida. Nesse sentido, recomenda-se estudos mais aprofundados relacionados aos hábitos e avaliação da qualidade de vida dos centenários, principalmente identificando os fatores de risco entre homens e mulheres. Acreditase que esta pesquisa permitiu ajudar a conhecer o perfil de mortalidade dos centenários em Curitiba e esperase que esse estudo possa contribuir para nortear futuras ações voltada para a população de pessoas com 100 anos ou mais.

\section{REFERÊNCIAS}

1. BRASIL. Estatuto do Idoso. 2009. Disponível em: https://bvsms.saude.gov.br/bvs/publicacoes/estatuto_idoso_2ed.pdf. Acessado em: 2 de setembro de 2021.

2. CAMARGOS MCS, GONZAGA MR. Viver mais e melhor? Estimativas de expectativa de vida saudável para a população brasileira. Cadernos de Saúde Pública, 2015; 31 (7): 1460-1472.

3. CAMPOS VS. Saúde da mulher idosa: resolubilidade do cuidado na estratégia saúde da família. Brazilian Journal of Development, 2020; 6 (12): 98787-98802.

4. CAPRIO AJ. Palliative care: renaming as supportive care and integration into comprehensive cancer care. CMAJ, 2016; 188 (10): 711-712.

5. CARVALHO MHR, et al. Tendência de mortalidade de idosos por doenças crônicas no município de Marília-SP, Brasil:. Epidemiologia e Serviços de Saúde, 2014; 23 (2): 347-354.

6. CEZAR-VAZ MR, et al. Skin cancer in rural workers: nursing knowledge and intervention. Revista da Escola de Enfermagem da USP, 2015; 49 (4): 0564-0571. 
7. CHEN Y-C, et al. Where and how centenarians die? The role of hospice care. American Journal of Hospice and Palliative Medicine $\AA$, 2019; 36 (12): 1068-1075.

8. EVANS CJ, et al. Place and cause of death in centenarians: a population-based observational study in England, 2001 to 2010. PLoS Med, 2014; 11 (6): e1001653.

9. GAUDIANO J, et al. Descripción de una población de pacientes portadores de enfermedad pulmonar intersticial asistidosenel Hospital Pasteur de Montevideo, Uruguay. Revista Uruguaya de Medicina Interna, 2020; 5 (2): 9-16.

10. INSTITUTO NACIONAL DO CÂNCER (INCA). Estimativa 2020: incidência de câncer no Brasil. 2020. Disponível em https://www.inca.gov.br/estimativa/introducao. Acessado em: 3 de novembro de 2020.

11. INSTITUTO PARANAENSE DE DESENVOLVIMENTO ECONÔMICO E SOCIAL (IPARDES). Projeção da População dos Municípios do Paraná para o Período 2018 a 2040 . Disponível em: http://www.ipardes.pr.gov.br/sites/ipardes/arquivos_restritos/files/documento/201909/nota_tecnica_populacao_projetada.pdf. Acessado em: 15 de setembro de 2021.

12. MARCUCCI FCI, CABRERA MAS. Morte no hospital e no domicílio: influências populacionais e das políticas de saúde em Londrina, Paraná, Brasil (1996 a 2010). Ciência \& Saúde Coletiva, 2015; 20 (3): 833-840.

13. MARTINS SC, et al. Centenários num Serviço de Medicina Interna: Casuística de 11 Anos. Medicina Interna, $2018 ; 25$ (2): 95-99.

14. MAXIMIANO-BARRETO MA, et al. A feminização da velhice: uma abordagem biopsicossocial do fenômeno. Interfaces Científicas-Humanas e Sociais, 2019; 8 (2): 239-252.

15. MOURA ECD, et al. Mortalidade no Brasil segundo perspectiva de gênero, anos 2000 e 2010. Revista Brasileira de Epidemiologia, 2016; 19 (2): 326-338.

16. NEPOMUCENO MR, TURRA CM. The population of centenarians in Brazil: historical estimates from 1900 to 2000. Population and Development Review, 2020; 46 (4): 813-833.

17. OLIVEIRA GMM, et al. Estatística Cardiovascular - Brasil 2020. Arquivos Brasileiros de Cardiologia, $2020 ; 115$ (3): 308-439.

18. OLIVEIRA TC, et al. Diferenciais de mortalidade por causas nas faixas etárias limítrofes de idosos. Rev. Bras. Geriatr. Gerontol., 2015; 18 (1): 85-94.

19. ORGANIZAÇÃO MUNDIAL DA SAÚDE (OMS). Relatório Mundial De Envelhecimento e Saúde. 2015. Disponível em: https://sbgg.org.br/wp-content/uploads/2015/10/OMS-ENVELHECIMENTO-2015-port.pdf. Acessado em: 24 de abril de 2020.

20. REIS CS, TURRA CM. Distribuição espacial dos centenários no Brasil: uma análise exploratória da qualidade dos dados dos censos de 2000 e 2010. Revista Espinhaço UFVJM, 2016; 5 (1): 52-61.

21. RIBEIRO TS, et al. Tendência temporal da mortalidade em idosos em municípios no estado do Acre, Revista Brasileira de Geriatria e Gerontologia, 2020; 23 (3): e200018.

22. ROBINE JM, CUBAYNES S. Worldwide demography of centenarians. Mechanisms of Ageing and Development, 2017 ; 165 (Pt B): 59-67.

23. XU J. Mortality among centenarians in the United States, 2000-2014. Hyattsville: National Center for Health Statistics, 2016; (233): 1-8.

24. YU R, et al. Trend of centenarian deaths in Hong Kong between 2001 and 2010. Geriatrics \& gerontology international, 2017; 17 (6): 931-936. 